\title{
Faulty Device Connector/Header
}

National Cancer Institute

\section{Source}

National Cancer Institute. Faulty Device Connector/Header. NCI Thesaurus. Code C100035.

There is a faulty connector/header in the device. (ACC) 